Courrier du Centre international Blaise Pascal

\title{
État des lieux pour mémoire
}

\section{Dominique Descotes}

\section{OpenEdition \\ Journals}

Édition électronique

URL : http://journals.openedition.org/ccibp/488

DOI : $10.4000 /$ ccibp.488

ISSN : 2493-7460

\section{Éditeur}

Centre international Blaise Pascal

\section{Édition imprimée}

Date de publication : 15 décembre 2008

Pagination : 41-42

ISBN : 978-2-84516-406-2

ISSN : 0249-6674

\section{Référence électronique}

Dominique Descotes, «État des lieux pour mémoire », Courrier du Centre international Blaise Pascal [En ligne], 30 | 2008, mis en ligne le 30 novembre 2015, consulté le 15 septembre 2020. URL : http:// journals.openedition.org/ccibp/488

Ce document a été généré automatiquement le 15 septembre 2020

Centre international Blaise Pascal 


\section{État des lieux pour mémoire}

\section{Dominique Descotes}

1 Le Courrier du CIBP n'a jamais eu de numéro 1. L'enquête lancée pour sa fondation le 16 juin 1979 ne portait pas de numéro, ce qui allait de soi, puisque nous y posions publiquement la question de son utilité et de la nécessité de fonder un centre pascalien. La livraison de 1980, qui publia les réponses au questionnaire diffusé l'année précédente, fut donc le $\mathrm{n}^{\circ} 2$. La présente livraison du Courrier $d u$ Centre international Blaise Pascal porte le numéro 30. Il n'était nullement certain, lorsque le projet d'un centre de documentation consacré à Pascal a été formé dans la suite du colloque Méthodes chez Pascal, que nous parviendrons à ce millésime. Il n'est pas inutile aujourd'hui de faire le point.

2 Les initiateurs de l'entreprise étaient parisiens, Jean Mesnard et Philippe Sellier, et clermontois, Thérèse Goyet et moi-même, alors tout récent assistant à la Faculté des Lettres. Par la suite, Antony McKenna, Gérard Ferreyrolles, Laurent Thirouin et d'autres sont venus étoffer l'équipe. Cette bande des quatre initiale était soutenue par le personnel directeur de la Bibliothèque municipale et interuniversitaire, savoir MarieThérèse Sart, conservateur en chef, Suzanne Montagne et Dominique Seguin. Pierre Cabanes, Président de l'Université Clermont II, et Roger Quilliot, sénateur maire de Clermont-Ferrand, avaient fait part de l'intérêt qu'ils accordaient au projet. Il fallait bien de tels patronages et surtout l'assurance et l'énergie dont fit toujours preuve Thérèse Goyet, alors professeur à la Faculté des Lettres, pour donner corps au projet : le peu de moyens dont bénéficiait au départ le Centre ne l'empêcha pas de lancer la "société CIBP» en cherchant des «actionnaires» qui devaient être aussi «fondateurs ». Le centre avait du reste un toit, la BMIU, et, grâce à la ville de Clermont, une bibliothécaire pour veiller sur le fonds pascalien, Caroline Durand d'abord, à laquelle ont succéder Claude Perrot, puis Véronique Pradier, grâce à laquelle le site de la Bibliothèque de Clermont comprend un catalogue électronique du fonds pascalien, aujourd'hui en développement.

3 Ses objectifs avaient à l'origine une dimension spécifiquement auvergnate : il s'agissait de repérer tout ce qui pouvait retracer l'histoire de la famille et des amis, notamment Domat, pour former une « physionomie régionale de Pascal ». Il devait aussi effectuer le 
recensement méthodique du fonds pascalien, à la Bibliothèque même, mais aussi aux archives départementales, sur l'intérêt desquelles Jean Mesnard attirait l'attention dans un article auquel fit écho, vingt ans plus tard, la Chronique des Pascal publiée à la librairie Champion par Régine Pouzet. Le CIBP peut alléguer quelques beaux résultats dans la découverte des documents pascaliens : la révélation d'une seconde pascaline qui fut acquise pour les musées de la ville, l'achat du manuscrit "Usage de la Machine », aujourd'hui propriété de la Bibliothèque, du testament d'Antoinette Fontfreyde, de la quittance signée de Pascal pour permettre à un jeune auvergnat d'aller faire son apprentissage à Paris, de la liste manuscrite inédite des religieuses de Port-Royal. L'enquête indiquait en effet l'importance que les correspondants accordaient à la fonction d'acquisition : Le CIBP devait participer à l'enrichissement de la bibliothèque en éditions anciennes et nouvelles, et collecter les publications nouvelles. Thérèse Goyet a conçu cette fonction d'acquisition dans un esprit original, à laquelle nous sommes demeurés fidèles : le CIBP, véritable organe intégré de la Bibliothèque, ne devait rien posséder en propre : tout document, tout ouvrage, du livre ancien au tiré-àpart, se trouvait donné en toute propriété à la Bibliothèque, c'est-à-dire à la collectivité. Le centre peut ainsi se prévaloir d'une fonction de service public qui fait sa spécificité. En retour il a servi souvent de fonds documentaire, par exemple pour la réalisation $\mathrm{du}$ grand dictionnaire de Port-Royal publié chez Champion par Antony McKenna et Jean Lesaulnier. Cette fonction le met en situation propice pour remplir la seconde mission qui lui était assignée par les réponses à l'enquête de fondation : l'information scientifique et documentaire. Recevant les productions de ses membres et correspondants, il se trouvait en mesure de diffuser dans le réseau des pascalisants l'annonce de leur publication.

4 L'enquête de fondation signalait aussi l'intérêt des correspondants pour la création d'un bulletin de liaison, annuel au moins, adapté aux besoins des chercheurs et des amis de Pascal, dans lequel l'information sur l'état, l'actualité et les progrès de la recherche sur Pascal occuperait la première place, à côté de la publication d'études de fond. Le Courrier a publié plusieurs documents inédits: le manuscrit «Usage de la machine ", acquis par le CIBP pour la Bibliothèque ( $\left.n^{\circ} 8,1986\right)$, par Guy Mourlevat, l'opuscule burlesque intitulé La folie du vide (1648), par Jacqueline Plantié et Jean Mesnard $\left(n^{\circ} 9,1987\right)$, plusieurs actes et relatifs à la famille Pascal, les clichés de la reconstitution de l'expérience du vide dans le vide par l'équipe de chercheurs japonais qui entoure K. Koyanagi $\left.\left(n^{\circ} 11\right), 1989\right)$. Pour l'information sur la recherche de pointe, plusieurs articles de Pol Ernst ont paru dans le Courrier avant la soutenance de sa thèse, révélant des travaux qui ont considérablement influé sur les recherches de ces dernières années. Annie Barnes et Hugo Friedrich figurent aussi au sommaire du Courrier. Enfin, certains numéros ont été consacrés à l'édition de colloques organisés par le centre, sur les Pensées, les Provinciales et la Raison des effets (ce dernier dirigé par M. Pécharman-Petit), à l'évocation de la maison de Pascal et à la commémoration de la nuit du Mémorial en l'église Saint-Jacques-du-Haut-Pas.

Enfin, nous avons consacré une part importante du Courrier à la publication des séances du séminaire sur les Pensées de Pascal, coorganisé par Laurent Thirouin (Lyon II), Antony McKenna (Saint-Etienne) et moi-même. La conception de ce séminaire est assez spéciale : les séances, qui se tiennent quatre fois l'an, avec deux séances à Clermont et deux à Lyon, font l'objet d'un compte rendu polycopié diffusé aux personnes qui le demandent; une fois les remarques recueillies et examinées, elles sont placées sur le site du CERHAC, en vue d'attirer d'autres observations. La publication dans le Courrier, 
après une dernière révision, est la dernière étape du travail. Cette opération, qui dure déjà depuis plus de six ans, est directement liée à la préparation de l'édition électronique des Pensées, dont nous décrivons le projet dans la présente livraison, projet actuellement rendu possible par l'engagement de Gilles Proust, ingénieur de recherche au CERHAC.

Durant ces trente années d'existence, le centre a considérablement évolué. Il a toujours conservé sa double tutelle de la Ville de Clermont-Ferrand et de l'Université Clermont II, aujourd'hui Université Blaise Pascal. Mais la constitution du Centre d'Études sur les Réformes, l'Humanisme et l'Âge Classique, lui a permis d'implanter une équipe de recherche dans un organisme qui s'est intégré lui-même à l'UMR 5037 du CNRS, important réseau qui réunit les universités de Lyon II, de Saint-Étienne et de Clermont, et l'École normale supérieure de Lettres de Lyon. Antony McKenna, auquel vient de succéder Pierre-François Moreau, a su donner la solidité nécessaire à cette UMR qui place le CIBP au cœur d'un puissant réseau de communication. Grâce à la localisation du CERHAC dans la Maison de la Recherche, nous avons pu créer une collection qui compte plusieurs ouvrages. Mais l'histoire n'est pas terminée: l'avenir s'annonce porteur de nouveaux changements, notamment avec la transformation de la Maison de la Recherche de Clermont-Ferrand en Maison des Sciences de l'Homme (dirigée par L. Jaffro), et avec les projets de transformation de la Bibliothèque de Clermont en Grande Bibliothèque.

7 Les activités du CIBP l'ont conduit à créer des contacts avec de nombreuses institutions, soit pour l'organisation de séminaires et de colloques. La coopération avec la société des Amis de Port-Royal et avec l'université de Catane en sont un exemple récent. Mais il faut aussi tenir compte de la constitution progressive des réseaux de pascalisants des pays étrangers, qui créent leur propre dynamique de recherche: on sait le poids du Japon dans les études pascaliennes; mais l'Italie est actuellement l'un des milieux les plus actifs ; le Brésil, la Roumanie, la Corée ont aussi donné naissance à des réseaux qui ne demandent qu'à prospérer. Ce réseau ne saurait évidement vivre sans l'association des Amis et Correspondants du CIBP, présidée par Jean Fau, qui assure le lien avec les amis de Pascal et l'animation en Auvergne, notamment par le biais de la Lettre aux Provinciaux. Il est de la responsabilité des membres "actionnaires", comme disait Thérèse Goyet, d'amener au CIBP les nouveaux membres qui feront son avenir.

\section{INDEX}

Mots-clés : Courrier du Centre International Blaise Pascal 